\title{
Possible Correlation between Severity of Retinopathy and Blood Levels of miRNAs (-146, -21 and 34a) in Type 2 Diabetic Patients
}

\author{
Hend G. Helal $^{\text {a }}$, Omnia A. Abdullah ${ }^{\mathrm{b}}$, Tamer I. Salem ${ }^{\mathrm{a}}$, Ahmed Daifalla ${ }^{\mathrm{a}}$
}

\begin{abstract}
a Department of ophthalmology, Benha faculty of medicine, Benha University, Egypt. b Department of Medical Biochemistry and Molecular Biology, Faculty of Medicine, Benha University, Egypt

Correspondence to: Hend G. Helal, Department of ophthalmology, Benha faculty of medicine, Benha University, Egypt.
\end{abstract}

Email:

hendhela175@gmail.com

Received: 20 March, 2020

Accepted: 10 November, 2020

\begin{abstract}
:
Background: Diabetic Retinopathy (DR), a leading cause of acquired visual loss, is a microvascular complication of DM, among the middle-aged and economically active population. At present, there is a lack of accuracy and specificity in our ability to identify this particular cohort. As the disease remains asymptomatic until the pathology is significantly advanced, screening to detect it during the early stages is necessary. Ability of miRNAs to alter or fine tune the expression of key regulators in various physiological processes and pathophysiological disease states makes them novel targets for diseases such as diabetic complications. Aim: The present work aimed to find out the possibility to use the expression pattern of miRNAs (146a, 21, and 34a) as biomarkers for early detection of DR in addition to the underling mechanisms. Also, to evaluate the correlation between them in each stage of DR to help in diagnosis by simple noninvasive methods. Patients and methods: Eighty patients with type 2 diabetes mellitus were involved in this study and diagnosed after investigation into normal, mild, moderate, severe NPDR and
\end{abstract} PDR patients. miRNAs 1436a,-21 and 34a were evaluated in the serum of patients in each group. Results: The present study revealed that the levels of miRNA-146 and miRNA -21 are increased in serum of DR patients in direct relation with the severity. However, serum miRNA-34a is decreased with progression of the disease. Conclusion miRNAs level in the serum of diabetic patients can be used as diagnostic biomarkers of DR.

Key words: Diabetic retinopathy, miRNAs, Diabetes mellitus 



\section{Introduction}

Diabetic Retinopathy (DR) is a microvascular complication of diabetes mellitus implicated in vision loss in adults (1). It is ranked as the fifth most common cause of moderate to severe visual impairment ${ }^{(2)}$. The retinal neurovascular unit is affected during diabetic retinopathy with subsequent disruption in the dynamic response that regulates oxygenation and nutrition of the neutrophil through blood flow and integrity of the BRB (3). Maintenance of retinal homeostasis by the vasculature is essential for exchange of nutrients, oxygen and metabolites between the neutrophil and the circulation to ensure retinal functions ${ }^{(4)}$.

The demand for photoreceptors is so high as the highest levels of oxygenation is in the choroid $^{(5)}$. The increased aerobic metabolism by photoreceptor inner segments under dark adaption conditions may require oxygen supplied by the retinal vasculature in order to maintain homeostasis. Although hyperglycemia has been demonstrated to initiate the pathology of DR, appreciable evidence suggests that oxidative stress and inflammatory changes in the retina play critical steps in the pathogenesis of the hyperglycemia-induced retinopathy (6). Also, pro-inflammatory lipids $^{(7)}$,epigenetic and epigenomic modifications ${ }^{(8)}$ insulin dysregulation and $\beta$-cellulin signaling ${ }^{(9)}$, which initiate and contribute to DR pathogenesis independently from hyperglycemic condition, have been identified

Oxidative stress has been implicated in the pathogenesis of DR either directly by oxidative damage to cells by reactive oxygen species (ROS)or indirectly via upregulation of ROS can lead to stimulation of inflammation and increased activation of $\mathrm{NF}-\kappa \mathrm{B}$, which, in turn, increased the release of pro-inflammatory cytokines and nitric oxide (NO).

Oral administration of antioxidants or genetic overexpression of superoxide dismutase ${ }^{(10)}$ has been shown to inhibit the diabetes- induced degeneration of retinal capillaries in animal studies. In addition, the inhibition of superoxides also inhibited glucose-induced release of pro-apoptotic cytochrome $\mathrm{c}$ and $\mathrm{Bax}$ in retinal pericytes and endothelial cells, thus further substantiating a causal link between mitochondrial oxidative stress and DR pathogenesis ${ }^{(11)}$. Under hyperglycemic and hypoxic conditions NADPH oxidase (Nox) 
enzymes are overexpressed in retinal endothelial cells and vessels leading to elevated ROS with subsequent DR pathogenesis. $^{(12)}$ Reducing several Nox isoform levels has been shown to alleviate several symptoms of DR, such as retinal neovascularization, blood retinal barrier breakdown, and leukostasis. Increasing evidence implicates inflammation has great rule in the pathogenesis of DR. this is proved by increase activation of many of the inflammatory mediators in DR, but the signaling involved in initiating this response is less clear.

Therefore diabetes-induced vascular pathology in animal models of DR can be improved by inhibition of proinflammatory enzymes or deletion of such enzymes ${ }^{(13)}$. Also the dramatic effect of corticosteroids on DME is another strong evidence the important role of inflammation in DR pathogenesis (14). Studies also have shown that leukocytes play an important role in the structural and functional abnormalities that characterize DR ${ }^{(15)}$, the specific cell types that mediate these pro-inflammatory effects are of great interest.

The clinical grading of diabetic retinopathy consists of mild and moderate non- proliferative diabetic retinopathy (background diabetic retinopathy), severe non-proliferative retinopathy (preproliferative diabetic retinopathy) and nonhigh risk and high risk proliferative diabetic retinopathy (proliferative diabetic retinopathy) (16). Typical clinical appearances Capillary micro aneurysms and a few intra retinal dot and blot hemorrhages can indicate mild, nonproliferative which can become evident years after the onset of T1DM but are often seen at the time of diagnosis in T2DM ${ }^{(17)}$.

The progress to either moderate or severe is mainly depend on increase in lesion density in addition to other signs reflecting capillary leakage, in the form of edema and intra retinal hard exudates, or signs reflecting capillary occlusion associated with ischemic damage of the nerve fiber layer, seen as cotton wool spots, venous dilation and beading, and intraretinal microvascular abnormalities ${ }^{(17)}$.

The risk of progression to proliferative DR is proportional to severity; $5 \%$ of mild nonproliferative DR progress to proliferative DR within 1 year, compared with $20 \%$ of moderate and $50 \%$ of severe nonproliferative DR. DME is separately classified as mild, moderate and severe and 
may occur even in mild, non-proliferative $\mathrm{DR}^{(18)}$.

Identifying dysregulated miRNAs, and an in-depth understanding of their mechanisms of actions and functional roles would provide better insights for the development of new biomarkers and therapeutic targets There is heightened interest in evaluating miRNAs as potential biomarkers for various diseases especially due to improvements in the technologies for miRNA detection in vitro and in vivo.

These include quantitative PCRs, microarrays and high throughput deep sequencing ${ }^{(19)}$. miRNAs are endogenously produced short non-coding RNAs of about 20-22 nucleotides in length that have been shown to play a key role in mammalian post-transcriptional gene expression ${ }^{(20)}$.

They are readily detectable in plasma and urine and their stability in these biofluids make them ideal candidate biomarkers for non-invasive and much needed early detection of diabetic complications. This is critical since such early detection can enhance clinical management, improve long term outcomes and greatly increase quality of life. Emerging evidence has emphasized the idea that circulating miRNAs in the plasma can be utilized as sensitive biomarkers for diseases like cancer, tissue injury and heart failure ${ }^{(21)}$. In last few years, miRNA levels in the urine, urinary sediment and circulating miRNA levels have been examined in patients with kidney disease ${ }^{(22)}$. Certain miRNAs such as192 and 205 were significantly higher in sera obtained from patients with primary focal segmental glomerulosclerosis and correlated with proteinuria and interstitial fibrosis ${ }^{(23)}$.

Studies in streptozotocin diabetic rats have suggested that miR-29b may have a protective role against the apoptosis of retinal ganglion cells and cells of the inner nuclear layer of retinas ${ }^{(24)}$. In the pathogenesis of diabetic retinopathy, increased oxidative stress is considered to play a major role ${ }^{(25}$ ) and studies have shown that the control of oxidative stress prevents epigenetic changes.

Thus, it is plausible that increased oxidative stress could be one of the mechanisms responsible for modulating epigenetic modifications in diabetic retinopathy. As reported, due to epigenetic modifications of various genes and transcription factors, the expression of the targeted genes is altered resulting in mitochondrial dysfunction, and ultimately, 
in the development of diabetic retinopathy (26)

The major focus of the clinical management of diabetes is not only glycemic control but also the prevention or reduction of debilitating complications. Combinatorial therapy with anti-miRNA and conventional drugs, or miRNA targeting alone could be a new approach ${ }^{(27)}$

The consequences of hyperglycemia result in deleterious changes in the expression patterns of various inflammatory and other pathologic genes and proteins which contribute to the development of vascular complications in diabetes ${ }^{(28)}$.

Circulating miRNAs have emerged as novel biomarkers of diabetes. Cells release miRNAs into the circulation or their surrounding environment, where miRNAs have a long lifespan of more than 2 weeks. Hirota et al. (29) first investigated the expression of 168 miRNAs in the vitreous humor and serum samples of patients with PDR and macular hole. This study found that six miRNAs (miR-15a, miR-320a, miR-320b, miR-93, miR-29a, and miR423-5p), related to angiogenesis and fibrosis, are significantly overexpressed in PDR ${ }^{(30)}$.
miRNA-146a expression has been studied in the kidney, retina, and sciatic nerve from both patients with diabetes microvascular changes and experimental diabetic animals. Results are conflicting with some studies reporting an increase and other a reduction in miRNA-146a expression ${ }^{(31)}$. This likely reflects variability in the compensatory anti-inflammatory miR-146a response in various stages and models of DMC. It has been reported thatmiRNA146a is an inhibitor to inflammatory process ${ }^{(32)}$.

It has been reported that, miRNA-21 is overexpressed (and contributes to the pathogenesis of DR by enhancing inflammation (33). Indeed, intervention studies have shown that both miRNA-21 deletion and intravitreal injection of a miRNA-21 inhibitor ameliorated retinal leakage and inflammation at least in part via upregulation of peroxisome proliferator-activated receptor- $\alpha$ (PPAR$\alpha)^{(33)}$, which is a miRNA-21 target and inhibits $\mathrm{NF}-\kappa \mathrm{B}$ by upregulating $\mathrm{I} \kappa \mathrm{B}-\alpha$. However, PPAR- $\alpha$ has also other beneficial effects particularly on metabolism that may explain its protective effect. Both upregulation and release of miRNA-21 appears to play a key role in sensory neuron-macrophage communication after damage to the peripheral nerve ${ }^{(34)}$. 
Qing et al. performed a differential miRNA profiling of serum samples from subjects with DR and identified 3 miRNAs (miRNA-21, miRNA-181c, and miRNA1179 significantly increased in patients with $\mathrm{PDR}^{(35)}$. A rise in plasma miRNA-21 levels in patients with type 2 diabetes and DR has also been confirmed by another study. These data are in line with studies showing miRNA-21 upregulation in experimental $\mathrm{DR}^{(36)}$. The up-regulation of miRNA-21 and miRNA-155 is NF- $\kappa$ B responsive in RECs and retinas of diabetic rats. Another study found that inhibition of miRNA-21significantly enhances the HGinduced endothelial cytotoxicity.

Further, overexpression of miRNA-21 inhibits the expression of death domain associated protein (DAXX), a proapoptotic mediator, whereas silencing DAXX mRNA reverses the inhibitory effects of miRNA21 on HG-induced endothelial apoptosis. This indicates that miRNA-21 can protect ECs from apoptosis by inhibiting the expression of DAXX ${ }^{(37)}$. It was found that increased levels of miRNA-21 in the vitreous are associated with retinal fibrosis, including PDR and proliferative vitreoretinopathy (PVR). The expression of miRNA-21 is enhanced and induced by
TGF- $\beta$, suggesting that its role positively correlates with disease progression ${ }^{(38)}$.

Kovacs and colleagues found several miRNA signatures for the upregulation of the transcription factor nuclear factor (NF)$\mathrm{kB}$, vascular endothelial growth factor (VEGF), and p53, reflecting the pathologic alterations of retinopathy ${ }^{(39)}$. They also indicated that the miRNA-34 family is upregulated in diabetic rats and some of them are important markers in the retina. Another study indicated the involvement of miR-34a in the proliferation and migration of retinal pigment epithelial (RPE) cells, important in vitreoretinopathy ${ }^{(37)}$.

Although the exact role of miRNA-34a in diabetic retinopathy does not fully investigated, it has been shown that miRNA-34a is a translational suppressor of SIRT1 ${ }^{(40)}$ and its function has been implicated in vascular senescence ${ }^{(41)}$. Altered expression of miRNA-34a is evident in several human pathologies, including cancer and cardiovascular disease ${ }^{(42)}$. Both, experimental and human diabetes are associated with increased expression of miRNA-34a, which in turn is linked to hyperglycemia-induced vascular dysfunction, oxidative stress. It has been reported that the gene encoding 
microRNA-34a is a direct transcriptional target of $\mathrm{p} 53^{(43)}$.

Therefore, it may be important to find out the relation between the level of expression of miRNAs and the severity of diabetic retinopathy to be either diagnostic and/or prognostic tools during management of DR.

\section{Patients and methods}

\section{Design of the work}

After diagnosis of diabetic patients (80) who were eligible to the study according to inclusion and exclusion criteria they were placed according to the severity of DR into the following groups: Normal fundus group, diabetic patients with no fundus abnormalities $(n=15)$; mild group, diabetic patients with mild fundus changes $(n=15)$; moderate group: diabetic patients with moderate changes in the fundus $(n=15)$; severe NPDR group, diabetic patients with non-proliferative severe changes in the fundus $(\mathrm{n}=15)$, and PDR group: diabetic patients with proliferative changes in the fundus $(n=20)$.From each patient in each group $6 \mathrm{ml}$ blood was taken and centrifuged to separate the sera that kept at$80{ }^{\circ} \mathrm{C}$ till used for biochemical and molecular analysis including lab investigations of blood glucose, lipid profile, serum creatinine. Also, miRNAs were isolated, quantified and tested for purity before evaluation of relative expressions of miRNAs (146a, 34a and 21) using qPCR.

\section{Patients}

The present study is prospective observational study. It was carried out in Ophthalmology Department, Faculty of Medicine, Benha University according to ethics committee approval from August 2019 to February 2020. Eighty type 2 diabetic patients were conducted in the present work (35 males and 45 females).Their age ranged from (49-71 years old). Patients were diagnosed according to American Diabetes Association diagnostic criteria.

The duration of diabetes ranged from 2-12 years. The inclusion criteria were; type 2 diabetic patients, patients diagnosed as normal fundus, mild, moderate, severe non proliferative DR and those with PDR. However, exclusion criteria were; history of liver cirrhosis.malignancy or infection, Renal diseases, other eye diseases associated with retinal degenerations. 


\section{Methods}

\section{Biochemical parameters}

Peripheral blood samples were collected following a 12-hour fasting. Glycosylated hemoglobin (HbA1c), blood glucose, total cholesterol, and triglycerides levels were estimated by routine enzymatic methods (Spinreact). TC, LDL, HDL and TG were measured using standard enzymatic methods.

\section{Fundus examination:}

This was done in outpatient clinic of ophthalmology department of Benha university Hospitals. Examination of fundus was done by 3 specialized personnel to confirm diagnosis by indirect ophthalmoscope using $20 \mathrm{D}$ lens and by 90D

\section{Evaluation of miRNAs expression:}

Total RNA was isolated from human serum by the standard TRIzol® Reagent extraction method (cat\#15596-026, Invitrogen, Germany). Reverse transcription (RT) reaction. Then, the complete Poly(A)+ RNA isolated from human serum was reverse transcribed into cDNA in a total volume of $20 \mu \mathrm{l}$ using RevertAidTM First Strand cDNA Synthesis Kit (MBI Fermentas, Germany).
StepOne ${ }^{\text {TM }}$ Real-Time PCR System from Applied Biosystems (Thermo Fisher Scientific, Waltham, MA USA) was used to determine the human serum's cDNA copy number.

\section{Statistical analysis}

Data were analyzed with Graph Pad Prism 5 (GraphPad Software, Inc., La Jolla, CA, USA). Quantitative data are expressed as the mean \pm standard deviation. One-way analysis of variance (ANOVA) was used to evaluate differences in serum miRNA levels between groups with tukey-kramer post hoc-test.

\section{Results}

\section{Biochemical parameters}

Data in table (1) explain no significant difference level regarding total cholesterol (TC), high density lipoprotein(LDL), low density lipoprotein (LDL), Triglycerides (TG) or glycosylated hemoglobin (HbA1c)amongst all groups.

\section{Eye examination}

Figure (1) Show different grade of severity of diabetic retinopathy patients in eye fluorescein angiography of diabetic patients with normal fundus (Fig.1A). In mild group, fluorescein angiography of mild 

NPDR fundus in diabetic patients shows micro aneurisms .There is also evidence of dot hemorrhages which appeared as hypoflourscent dots (fig. 1B). Moderate NPDR group shows blocked fluorescence by dot and blot hemorrhages, leaking and non-leaking micro aneurisms and coexisting macular edema. (fig. 1C).
Severe NPDR (fig.1D) with extensive blot hemorrhages in all retinal quadrants and vascular abnormalities with evidence of multiple micro aneurisms and no evidence of neovascularization. In PDR patients (fig.1E) there is extensive leakage around disc from NVDs and /or NVEs with or without preretinal hemorrhages.

Table 1:

\begin{tabular}{lcccccc}
\hline Parameter & $\begin{array}{c}\text { Normal fundus } \\
(\mathbf{n}=\mathbf{1 5})\end{array}$ & $\begin{array}{c}\text { Mild NDPR } \\
(\mathbf{n}=\mathbf{1 5})\end{array}$ & $\begin{array}{c}\text { Moderate NPDR } \\
(\mathbf{n}=\mathbf{1 5})\end{array}$ & $\begin{array}{c}\text { Severe NRNP } \\
(\mathbf{n = 1 5})\end{array}$ & $\begin{array}{c}\text { PDR } \\
(\mathbf{n}=\mathbf{2 0})\end{array}$ & P value \\
\hline TC (mg/dL) & $179.04 \pm 70$ & $163.57 \pm 63.8$ & $189.87 \pm 73.47$ & $167.05 \pm 71.54$ & $184.84 \pm 68.83$ & $>0.05$ \\
HDL (mg/dL) & $44.47 \pm 12$ & $43.7 \pm 9.67$ & $46.02 \pm 8.51$ & $44.86 \pm 12.76$ & $43.31 \pm 8.89$ & $>0.05$ \\
LDL (mg/dL) & $102.09 \pm 25.91$ & $104.4 \pm 21.27$ & $97.83 \pm 27.07$ & $108.28 \pm 25.91$ & $102.86 \pm 20.88$ & $>0.05$ \\
TG (mg/dL) & $126.66 \pm 93$ & $128.43 \pm 106.29$ & $132.86 \pm 94.77$ & $147.03 \pm 126.66$ & $150.58 \pm 108.06$ & $>0.05$ \\
HbA1c & $7.62 \pm 1.68$ & $6.54 \pm 1.55$ & $7.71 \pm 1.85$ & $6.33 \pm 1.7$ & $7.8 \pm 1.66$ & $>0.05$ \\
\hline
\end{tabular}

serum level of total cholesterol (TC), high density lipoprotein(LDL), low density lipoprotein (LDL), Triglycerides (TG) and glycosylated hemoglobin (HbA1c)in different grade of diabetic retinopathy groups.
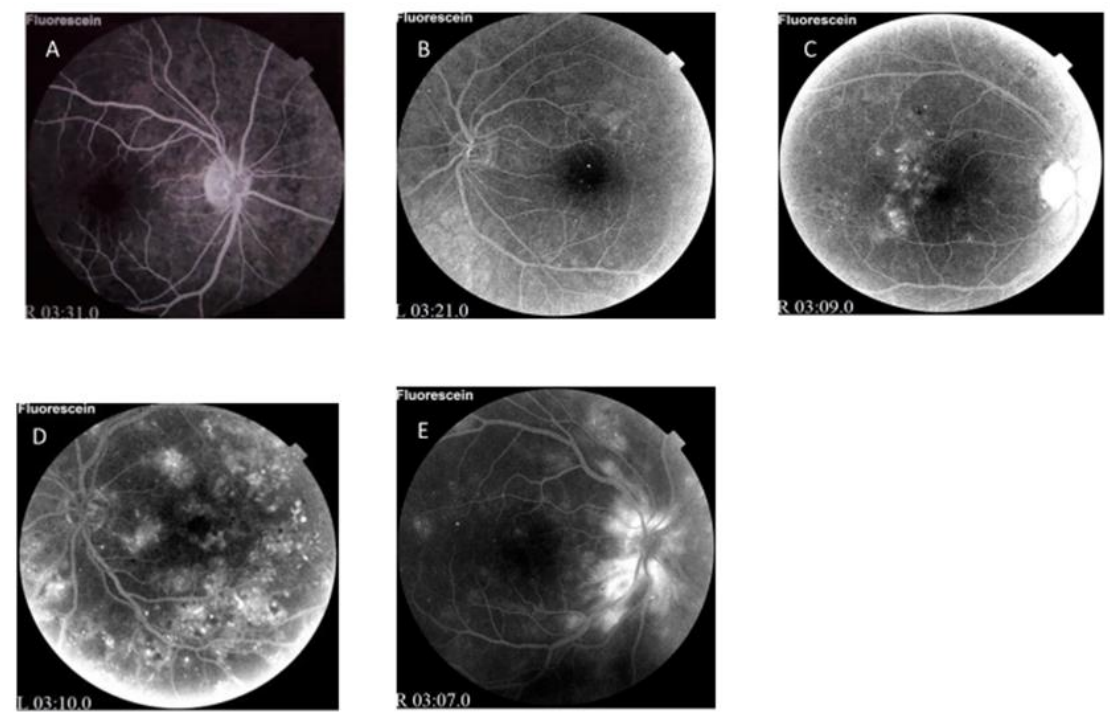

Figure (1): Different Stages of diabetic retinopathy

A: Normal, B: Mild NPDR, C: Moderate NPDR, D: Severe NPDR and E: PDR 



\section{Discussion}

In DR, early detection and treatment is of vital importance as it may prevent vision loss and blindness and screening for DR is cost-effective when compared with disability loss for people going blind in the absence of a screening program. The compliance for the screening program should be more than $80 \%$ for more gains (44). At present, effective treatments for DR exist and can prevent progression to blindness provided the disease is diagnosed early ${ }^{(45)}$.

The use of miRNAs as biomarkers may help in early diagnosis of DR. There is evidence that several miRNAs target specific mRNAs for regulating the progression of DR. By annealing to partially complementary sequences in the target mRNAs, miRNAs mediate translational repression or degradation of mRNAs, resulting in the downregulation of protein expression ${ }^{(46)}$.

The present study was designed to investigate the patterns of expressions of serum miRNAs (146a,21and 34a) in different stages of DR patients.

Data in present work revealed that there is direct relation between relative expression of miRNA-146a in the serum and severity of DR. This finding is strongly supported by the study of Feng et al. ${ }^{(47)}$ who explained that miRNA-146a exhibited increases in expression along with DR development and it is upregulated by time. Also, it has been reported that serum miRNA-146a was elevated in T2DM subjects compared to healthy controls ${ }^{(48)}$. In this regard, miRNA-146a has reported as an inhibitor to inflammatory process ${ }^{(49)}$. In addition, intravitreal injection of miR-146a ameliorated both microvascular leakage and retinal functional defects and reduced expression of intercellular adhesion molecule 1 (ICAM-1), a NF-kB downstream gene. Moreover, it has been found that there is an up-regulation of several NF-kB responsive miRNAs, including miR-146 in the retina and retinal endothelial cells (RECs) from Streptozotocin (STZ)-induced T1D rats ${ }^{(50)}$. Furthermore, it has been suggested that down-regulation of miRNA-146a could be a key mechanism for increased extracellular matrix protein production in diabetes ${ }^{(51)}$.

In the present study miRNA-21 expression in the serum of diabetic patients was increased along with increased severity of DR. miRNA-21 was increased in the 
vitreous humor of patients with proliferative DR, supporting that miRNA21 may play a role in DR ${ }^{(52)}$ In addition, diabetes-was reported to induce PPAR- $\alpha$ downregulation has been shown to play a key role in retinal inflammation in DR ${ }^{(53)}$. The authors explained that that overexpression of miRNA-21 is at least partly responsible for $\operatorname{PPAR} \alpha$ downregulation and retinal inflammation in DR.

Regarding miRNA-34a, the obtained data from the present work revealed that, diabetic patients with normal fundus show the highest level of serum miRNA-34a expression. This expression was declined with the progression of the disease and reaches its minimal level in diabetic patients with PDR.

These data can be supported by previous studies indicated increased expression of miRNA-34a in experimental and human diabetes, which in turn is linked to hyperglycemia-induced vascular dysfunction ${ }^{(54)}$, oxidative stress ${ }^{(55)}$, and apoptosis. Thounaojam et al. ${ }^{(56)}$ explained that increased oxidative/nitrative stress plays a causal role in retinal vascular dysfunction and hyperglycemia-induced premature senescence $^{(57)}$.
The decreased expression of miRNA-34a in the present work may be explained depending on the previous findings of the relation between increase oxidative stress and NF-kB activation and its effect on p53 activation (inducer of miRNA-34a expression). In this regard, p53 activation led to induction of the miRNA-34a, which sensitized beta cells to apoptosis and inhibited the insulin exocytosis pathway, resulting in impaired insulin secretion ${ }^{(58)}$. Also, Nesca et al. ${ }^{(59)}$ found that miRNA34a was significantly upregulated in the serum of patients with T2DM. In addition, an increase in miRNA-34a is associated with the activation of $\mathrm{p} 53$, and results in sensitization to apoptosis and impaired nutrient-induced secretion ${ }^{(58)}$. Moreover, expression of miRNA-34a is augmented by p53 in response to genotoxic stress, both in vitro and in vivo ${ }^{(60)}$.

\section{Conclusion}

Conclusively, circulating miRNAs can be used as diagnostic and prognostic tools for DR.In particular, miRNA-146a, miRNA-21 and miRNA-34a expression can help in early prediction of DR. In addition, the pattern of their serum expression together may add a new effective window for staging of the severity of DR. Furthermore, 
understanding their role in progression of DR can provide a new therapeutic window for protection against or attenuation of disease progression.

\section{References}

1- Fong, D. S., Aiello, L. P., Ferris, F. L., \& Klein, R. (2004). Diabetic retinopathy. Diabetes Care, 27(10): 2540-2554.

2-Bourne RR White RA, Smith JL, Flaxman SR, Price H, et al, S. G. A. (2013). Causes of vision loss worldwide, 1990-2010: A systematic analysis. Lancet Glob Health, 1(6): e339-49.

3-Klaassen, I., Van Noorden, C. J. F., \& Schlingemann, R. O. (2013). Molecular basis of the inner blood-retinal barrier and its breakdown in diabetic macular edema and other pathological conditions. Progress in Retinal and Eye Research, 34:19-48.

4-Kur, J., Newman, E. A., \& Chan-Ling, T. (2012). Cellular and physiological mechanisms underlying blood flow regulation in the retina and choroid in health and disease. Progress in Retinal and Eye Research, 31(5): 377-406.

5-Caprara, C., \& Grimm, C. (2012). From oxygen to erythropoietin: relevance of hypoxia for retinal development, health and disease. Progress in Retinal and Eye Research, 31(1):89-119.
6-Roy, S., Kern, T. S., Song, B., \& Stuebe, C. (2017). Mechanistic insights into pathological changes in the diabetic retina: implications for targeting diabetic retinopathy. The American Journal of Pathology, 187(1): 9-19.

7-Crosby-Nwaobi, R., Chatziralli, I., Sergentanis, T., Dew, T., Forbes, A., \& Sivaprasad, S. (2015). Cross talk between lipid metabolism and inflammatory markers in patients with diabetic retinopathy. Journal of Diabetes Research, 2015.

8-Kowluru, R. A., \& Mishra, M. (2015). Contribution of epigenetics in diabetic retinopathy. Science China Life Sciences, 58(6): 556-563.

9-Anand-Apte, B., Ebrahem, Q., Cutler, A., Farage, E., Sugimoto, M., Hollyfield, J., \& Folkman, J. (2010). Betacellulin induces increased retinal vascular permeability in mice. PLoS One, 5(10): e13444.

10-Kanwar, M., Chan, P.-S., Kern, T. S., \& Kowluru, R. A. (2007). Oxidative damage in the retinal mitochondria of diabetic mice: possible protection by superoxide dismutase. Investigative Ophthalmology \& Visual Science, 48(8): 38053811.

11-Kowluru, R. A., \& Abbas, S. N. (2003). Diabetesinduced mitochondrial dysfunction in the retina. Investigative Ophthalmology \& Visual Science, 44(12):5327-5334. 
12-Al-Shabrawey, M., Bartoli, M., El-Remessy, A. B., Ma, G., Matragoon, S., Lemtalsi, T., Caldwell, R. W., \& Caldwell, R. B. (2008). Role of NADPH oxidase and Stat3 in statin-mediated protection against diabetic retinopathy. Investigative Ophthalmology \& Visual Science, 49(7):32313238.

13-13- Tang, J., \& Kern, T. S. (2011). Inflammation in diabetic retinopathy. Progress in Retinal and Eye Research, 30(5):343-358.

14- Silva, P. S., Sun, J. K., \& Aiello, L. P. (2009). Role of steroids in the management of diabetic macular edema and proliferative diabetic retinopathy. Seminars in Ophthalmology, 24(2): 9399.

15-Talahalli, R., Zarini, S., Tang, J., Li, G., Murphy, R., Kern, T. S., \& Gubitosi-Klug, R. A. (2013). Leukocytes regulate retinal capillary degeneration in the diabetic mouse via generation of leukotrienes. Journal of Leukocyte Biology, 93(1): 135-143.

16-Wong, T.Y., Klein, R., Islam, F.M., Cotch, M.F., Folsom, A.R., Klein, B.E., Sharrett, A.R., \& Shea S.(2006). Diabetic retinopathy in a multi-ethnic cohort in the United States. American Journal of Ophthalmology, 141(3): 446-455.

17-Sim, D. A., Keane, P. A., Rajendram, R., Karampelas, M., Selvam, S., Powner, M. B., Fruttiger, M., Tufail, A., \& Egan, C. A. (2014).
Patterns of peripheral retinal and central macula ischemia in diabetic retinopathy as evaluated by ultra-widefield fluorescein angiography. American Journal of Ophthalmology, 158(1).

18-Antonetti, D. A., Klein, R., \& Gardner, T. W. (2012). Diabetic retinopathy. In New England Journal of Medicine, 366(13): 1227-1239.

19- Farazi, T. A., Spitzer, J. I., Morozov, P., \& Tuschl, T. (2011). MiRNAs in human cancer. Journal of Pathology ,223(2): 102-115.

20-Bartel, D. P. (2009). MicroRNAs: Target Recognition and Regulatory Functions. In Cell, 136(2): 215-233.

21- Fabbri, M. (2010). MiRNAs as molecular biomarkers of cancer. In Expert Review of Molecular Diagnostics, 10(4): 435-444.

22- Jiao, Y., Li, Y., Fu, Z., Hou, L., Chen, Q., Cai, Y., Jiang, P., He, M., \& Yang, Z. (2019). OGDHL Expression as a Prognostic Biomarker for Liver Cancer Patients. Disease Markers, 2019: 1-9.

23- Cai, X., Xia, Z., Zhang, C., Luo, Y., Gao, Y., Fan, Z., Liu, M., \& Zhang, Y. (2013). Serum microRNAs levels in primary focal segmental glomerulosclerosis. Pediatric Nephrology, 28(9): 1797-1801.

24-Silva, V. A. O., Polesskaya, A., Sousa, T. A., Corrêa, V. M. A., André, N. D., Reis, R. I., Kettelhut, I. C., Harel-Bellan, A., \& De Lucca, F. L. 
(2011). Expression and cellular localization of microRNA-29b and RAX, an activator of the RNAdependent protein kinase (PKR), in the retina of streptozotocin-induced diabetic rats. Molecular Vision, 17:2228-2240.

25-Santos,M., Mohammad,J., Zhong, G.Q., \& Kowluru, R.A. (2011). Diabetic Retinopathy, Superoxide Damage and Antioxidants. Current Pharmaceutical Biotechnology, 12(3):352-361.

26- Kowluru,R. A., Julia M. Santos,J.M., \& Manish, M.(2013). Epigenetic Modifications and Diabetic Retinopathy, BioMed Research International, 2013: 635284, 1-9.

27-Kato, M., Putta, S., Wang, M., Yuan, H., Lanting, L., Nair, I., Gunn, A., Nakagawa, Y., Shimano, H., Todorov, I., Rossi, J. J., \& Natarajan, R. (2009). TGF- $\beta$ activates Akt kinase through a microRNAdependent amplifying circuit targeting PTEN. Nature Cell Biology, 11(7): 881-889.

28- King, G. L. (2008). The Role of Inflammatory Cytokines in Diabetes and Its Complications. Journal of Periodontology, 79(8s):1527-1534.

29- Hirota, K., Keino, H., Inoue, M., Ishida, H., \& Hirakata, A. (2015). Comparisons of microRNA expression profiles in vitreous humor between eyes with macular hole and eyes with proliferative diabetic retinopathy. Graefe's Archive for Clinical and Experimental Ophthalmology, 253(3): 335-342.
30-Zampetaki, A., Willeit, P., Burr, S., Yin, X., Langley, S. R., Kiechl, S., Klein, R., Rossing, P., Chaturvedi, N., \& Mayr, M. (2016). Angiogenic microRNAs linked to incidence and progression of diabetic retinopathy in type 1 diabetes. Diabetes, 65(1): 216-227.

31-Liu, X. S., Fan, B., Szalad, A., Jia, L., Wang, L., Wang, X., Pan, W., Zhang, L., Zhang, R., \& Hu, J. (2017). MicroRNA-146a mimics reduce the peripheral neuropathy in type 2 diabetic mice. Diabetes, 66(12): 3111-3121.

32- Barutta,F; Bellini,S.; Mastrocola, R; Bruno,G\& Gruden,G. (2018). MicroRNA and Microvascular Complications of Diabetes. Int. J. Endocrinol. 2018: 6890501.

33-Chen, Q., Qiu, F., Zhou, K., Matlock, H. G., Takahashi, Y., Rajala, R. V. S., Yang, Y., Moran, E., \& Ma, J. (2017). Pathogenic role of microRNA21 in diabetic retinopathy through downregulation of PPAR $\alpha$. Diabetes: 66(6):1671-1682.

34-Simeoli, R., Montague, K., Jones, H. R., Castaldi, L., Chambers, D., Kelleher, J. H., Vacca, V., Pitcher, T., Grist, J., \& Al-Ahdal, H. (2017). Exosomal cargo including microRNA regulates sensory neuron to macrophage communication after nerve trauma. Nature Communications, 8(1): 1778.

35-Qing, S., Yuan, S., Yun, C., Hui, H., Mao, P., Wen, F., Ding, Y., \& Liu, Q. (2014). Serum miRNA 
biomarkers serve as a fingerprint for proliferative diabetic retinopathy. Cellular Physiology and Biochemistry, 34(5): 1733-1740.

36-Zou, H.-L., Wang, Y., Gang, Q., Zhang, Y., \& Sun, Y. (2017). Plasma level of miR-93 is associated with higher risk to develop type 2 diabetic retinopathy. Graefe's Archive for Clinical and Experimental Ophthalmology,255(6): 1159-1166.

complications. Diabetes, 60(11): 2975-2984.

37-Hou, Q., Tang, J., Wang, Z., Wang, C., Chen, X., Hou, L., Dong, X. Da, \& Tu, L. L. (2013). Inhibitory effect of microRNA-34a on retinal pigment epithelial cell proliferation and migration. Investigative Ophthalmology and Visual Science, 54(10): 6481-6488.

38-Jindal, V. (2015). Neurodegeneration as a primary change and role of neuroprotection in diabetic retinopathy. Molecular Neurobiology, 51(3): 878884.

39-Kovacs, B., Lumayag, S., Cowan, C., \& Xu, S. (2011). MicroRNAs in early diabetic retinopathy in streptozotocin-induced diabetic rats. Investigative Ophthalmology \& Visual Science, 52(7): 44024409 .

40-Tian, X.-F., Ji, F.-J., Zang, H.-L., \& Cao, H. (2016). Activation of the miR-34a/SIRT1/p53 signaling pathway contributes to the progress of liver fibrosis via inducing apoptosis in hepatocytes but not in HSCs. PLoS One, 11(7): e0158657.

41-Badi, I., Burba, I., Ruggeri, C., Zeni, F., Bertolotti, M., Scopece, A., Pompilio, G., \& Raucci, A. (2014). MicroRNA-34a induces vascular smooth muscle cells senescence by SIRT1 downregulation and promotes the expression of age-associated proinflammatory secretory factors. Journals of Gerontology Series A: Biomedical Sciences and Medical Sciences: 70(11): 1304-1311.

42-Li, N., Wang, K., \& Li, P.-F. (2015). MicroRNA34 family and its role in cardiovascular disease. Critical ReviewsTM in Eukaryotic Gene Expression, 25(4).

43-Raver-Shapira, N., Marciano, E., Meiri, E., Spector, Y., Rosenfeld, N., Moskovits, N., Bentwich, Z., \& Oren, M. (2007). Transcriptional activation of miR-34a contributes to p53-mediated apoptosis. Molecular Cell, 26(5): 731-743.

44-Dasbach, E. J., Fryback, D. G., Newcomb, P. A., Klein, R., \& Klein, B. E. (1991). Cost-effectiveness of strategies for detecting diabetic retinopathy. Medical Care, 29(1):, 20-39.

45- Aiello, L.P., Odia, I., Glassman, A.R., Melia, M., Jampol, L.M., Bressler,N.M., Kiss, S., et al. (2019). 46-Wu, J., Gao, Y., Ren, A., Zhao, S., Zhong, M., Peng, Y., Shen, W., Jing, M., \& Liu, L. (2012). Altered microRNA expression profiles in retinas 
with diabetic retinopathy. Ophthalmic Research, 47(4): 195-201.

47-Feng, B., Chen, S., McArthur, K., Wu, Y., Sen, S., Ding, Q., Feldman, R. D., \& Chakrabarti, S. (2011). miR-146a-mediated extracellular matrix protein production in chronic diabetes

48-Lee, H. W., Khan, S. Q., Khaliqdina, S., Altintas, M. M., Grahammer, F., Zhao, J. L., Koh, K. H., Tardi, N. J., Faridi, M. H., \& Geraghty, T. (2017). Absence of miR-146a in podocytes increases risk of diabetic glomerulopathy via up-regulation of ErbB4 and Notch-1. Journal of Biological Chemistry,292(2): 732-747.

49-Yousefzadeh, N., Alipour, M. R., \& Soufi, F. G. (2015). Deregulation of NF-кB-miR-146a negative feedback loop may be involved in the pathogenesis of diabetic neuropathy. Journal of Physiology and Biochemistry, 71(1): 51-58.

50-Kato, M., Park, J. T., \& Natarajan, R. (2012). MicroRNAs and the glomerulus. Experimental Cell Research, 318(9): 993-1000.

51- Liang, M., Liu, Y., Mladinov, D., Cowley Jr, A. W., Trivedi, H., Fang, Y., Xu, X., Ding, X., \& Tian, Z. (2009). MicroRNA: a new frontier in kidney and blood pressure research. American Journal of Physiology-Renal Physiology, 297(3): F553-F558. 52-Usui-Ouchi, A., Ouchi, Y., Kiyokawa, M., Sakuma, T., Ito, R., \& Ebihara, N. (2016).
Upregulation of Mir-21 levels in the vitreous humor is associated with development of proliferative vitreoretinal disease. PloS One, 11(6): e0158043.

53-Ding, L., Cheng, R., Hu, Y., Takahashi, Y., Jenkins, A. J., Keech, A. C., Humphries, K. M., Gu, X., Elliott, M. H., \& Xia, X. (2014). Peroxisome Proliferator-Activated Receptor $\alpha$ Protects Capillary Pericytes in the Retina. The American Journal of Pathology, 184(10): 2709-2720.

54-Zhao, D., Wang, N.-S., Chen, F., Li, Z.-B., Li, X.T., \& Zhu, X.-X. (2019). Intravenous Injection of miR-34a Inhibitor Alleviates Diabetes MellitusInduced Vascular Endothelial Dysfunction by Targeting NOTCH1. Experimental and Clinical Endocrinology \& Diabetes, 127(04): 255-262.

55-Banerjee, J., Khanna, S., \& Bhattacharya, A. (2017). MicroRNA regulation of oxidative stress. Oxidative Medicine and Cellular Longevity, 2017. 56-Thounaojam, M. C., Jadeja, R. N., Warren, M., Powell, F. L., Raju, R., Gutsaeva, D., Khurana, S., Martin, P. M., \& Bartoli, M. (2019). MicroRNA-34a (miR-34a) Mediates Retinal Endothelial Cell Premature Senescence through Mitochondrial Dysfunction and Loss of Antioxidant Activities. Antioxidants, 8(9): 328.

57-Wu, Y., Tang, L., \& Chen, B. (2014). Oxidative stress: implications for the development of diabetic 
retinopathy and antioxidant therapeutic perspectives. Oxidative Medicine and Cellular Longevity, 2014.

58-Lovis, P., Roggli, E., Laybutt, D. R., Gattesco, S., Yang, J.-Y., Widmann, C., Abderrahmani, A., \& Regazzi, R. (2008). Alterations in microRNA expression contribute to fatty acid-induced pancreatic $\quad \beta$-cell dysfunction. Diabetes, 57(10):2728-2736.
59-Nesca, V., Guay, C., Jacovetti, C., Menoud, V., Peyot, M.-L., Laybutt, D. R., Prentki, M., \& Regazzi, R. (2013). Identification of particular groups of microRNAs that positively or negatively impact on beta cell function in obese models of type 2 diabetes. Diabetologia, 56(10): 2203-2212.

60-Marsit, C. J., Eddy, K., \& Kelsey, K. T. (2006). MicroRNA responses to cellular stress. Cancer Research, 66(22): 10843-10848.

To cite this article: Hend G. Helal, Omnia A. Abdulla, Tamer I. Salem, Ahmed Daifalla. Possible Correlation between Severity of Retinopathy and Blood Levels of miRNAs (-146, -21 and 34a) in Type 2 Diabetic Patients. BMFJ 2020; 37 (surgical issue):89-105. DOI: $10.21608 / \mathrm{bmfj} .2020 .26237 .1230$ 